\title{
THE COUPLED DIFFUSION OF HEAT AND MOISTURE IN BULK WOOL
}

\author{
J.M. HEATH
}

A mathematical model for the problem of transfer and absorption of heat and moisture in bulk wool at humidities below saturation is discussed. In its full generality this model involves eight coupled nonlinear equations. Approximations can be made to this system, within a limited range of temperature and humidity, which depend on the size and density of the medium and the magnitude of the superficial velocity. Where the convective effects are negligibly small, and the local rate of transfer of moisture into individual fibres is large in comparison with the rate of transfer through the medium, the model can be reduced to a pair of coupled nonlinear diffusion equations of the form

$$
\frac{\partial u_{i}}{\partial t}=\sum_{j} a_{i j}(u) \nabla^{2} u_{j}, \quad i=1,2 .
$$

This model is the same as that derived by Henry [1] who obtains solutions by linearising the equations.

A treatment of this problem based on a boundary layer technique is presented, which allows the quasilinear system to be simplified without removing its essential nonlinear character. The result is two systems of equations (zero order terms in inner and outer expansions) which can be partially solved. The matching between the two allows prediction of pseudo equilibrium temperatures in agreement with observed values. One other result of the analysis is the derivation of a Fickian equation governing

Received 20 August 1982. Thesis submitted to Victoria University of Wellington, May 1982. Degree approved: August 1982. Supervisors: Dr A. $\mathrm{McNabb}$ and Dr G.C. Wake. 
the concentration of absorbed moistured.

The uniqueness of solution is established for the above system of equations, and also for a related semilinear system in which allowance is made for the finite rate of local mass transfer.

Upper and lower bounds for solutions of the linear version of the above system can be determined by first transforming the equations so that $A=\left(a_{i j}\right)$ is diagonal. The question of the existence of similar bounds for solutions of the quasilinear system is considered, but the analagous results can not be established.

Department of Mathematics,

Victoria University of Wellington, Private Gab, Well ington, New Zeal and. 